\title{
LOW CONTRAST VISUAL ACUITY CHANGES IN HUMAN IMMUNO-DEFICIENCY VIRUS (HIV) INFECTION
}

\author{
ERKAN MUTLUKAN, BALJEAN DHILLON, PETER ASPINALL, JAMES F. CULLEN \\ Edinburgh
}

\begin{abstract}
SUMMARY
A pilot study of low contrast visual acuity testing using Regan Charts has been undertaken in 34 patients seropositive for Human Immuno-Deficiency Virus and 20 normal control subjects. Low contrast visual acuities of the HIV (+) patients both with and without HIV retinopathy were found to be significantly lower than the age-matched controls $(p<0.01)$. This finding is probably attributable to pathology related to HIV in the visual pathways/Central Nervous System. Lowest contrast Chart (Chart C) was found to be a useful diagnostic tool for HIV retinopathy and presumed neuropathy.
\end{abstract}

A high percentage (40-94\%) of patients infected with Human Immuno-Deficiency Virus (HIV) develop ocular involvement at some stage during their disease. ${ }^{1}$ The visual disturbances which result from vascular and infectious involvement of the retina or from neuro-ophthalmic abnormalities of the central nervous system contribute to the morbidity of Acquired Immune Deficiency Syndrome (AIDS).

The highest rate of HIV infection in the UK is found in the Edinburgh, Lothian area with approximately one new case per week and with 130 known infected individuals per 100,000 of population. In the fast growing AIDS epidemic, a more subtle test than Snellen visual acuity may be of value in early detection of ocular and neurological involvement among these patients.

It has been shown that low contrast vision may be reduced while Snellen visual acuity remains normal in patients with early diabetic retinopathy, Parkinson's disease, glaucoma, and cerebral visual pathway disorders. $^{2,3,4}$ Low contrast visual acuity testing has been found to be more sensitive than pattern-evoked visual response testing for detecting visual dysfunction due to optic nerve lesion and $93 \%$ sensitive for detecting subtle optic neuropathies. ${ }^{5,6}$ Letter reading performance with the Regan Low Contrast Acuity Charts has also been shown to be comparable to sine-wave grating Contrast Sensitivity Function testing in detecting visual pathway abnormal-

Correspondence to: Dr. Erkan Mutlukan, Royal Infirmary of Edinburgh, Princess Alexandra Eye Pavilion, Edinburgh EH3 9HA, Scotland. ities. ${ }^{2,4,7}$ The very low contrast Regan optotype test chart has been found to be as sensitive as pattern-reversal visual evoked potentials in detecting visual dysfunction in recovered optic neuritis with near-normal visual acuity. ${ }^{8}$

We performed a pilot study of low contrast visual acuities in a group of HIV infected individuals to investigate the possible use of Low-Contrast Visual Acuity Charts in ophthalmic and neurological follow-up of such cases.

\section{PATIENTS AND METHOD}

The right eyes of 34 HIV sero-positive individuals, four females and 30 males, aged between 21 and 40 years (mean 29.3 years) were examined. Patients were selected randomly from medical clinics that provide care for HIV infected individuals. Two eyes had 6/9 visual acuity and the remaining 32 eyes had Snellen acuities of $6 / 6$ or better.

The right eyes of 20 sero-negative controls, aged between 25 and 39 years (mean 30 years) with no history of eye disease, were also tested. All had Snellen acuities of $6 / 6$ or better.

Each patient was tested using Regan Low Contrast Visual Acuity Charts as part of their ophthalmic examination. These are similar to Snellen charts but have both high contrast and reduced contrast letters. The test letters are presented as dark figures on a white background, and patient's performance depends on his/her ability of high, medium and low contrast visual spatial processing. As well as testing the visual acuity at each given contrast level, the charts facilitate investigation of contrast sensitivity at any given letter size. The three charts used were chart $\mathrm{A}$, with high contrast $(96 \%)$ which is equivalent to conventional acuity charts; chart $\mathrm{B}$, which is of intermediate (11\%) contrast; and chart C, a low (4\%) contrast chart. All charts were read monocularly from a distance of three meters under standardised illumination $\left(103 \mathrm{~cd} / \mathrm{m}^{2}\right)$.

A low contrast visual acuity score for each eye was calculated from the number of complete lines read correctly plus any fraction of the eight optotypes on the following line. This was done for each high, intermediate and low contrast visual acuity test chart.

Analysis of Variance was used for statistical evaluation.

\section{RESULTS}

Twenty-three of the 34 HIV positive patients had no 


\section{Retinal Involvement Among HIV Seropositive Subjects (n: 34)}

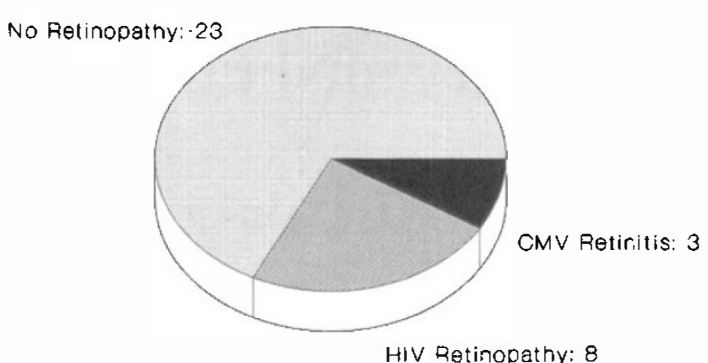

Fig. 1. Details of the HIV sero-positive population (34 patients) tested according to fundus appearance.

history of HIV related ocular involvement and no visible retinal or optic disc abnormalities. Eight of the remaining 11 patients had HIV retinopathy (cotton-wool spots) (Fig. 1). All these lesions were outside the macular area. Three patients had active cytomegalovirus retinitis (CMVR) affecting the peripheral retina, with Snellen visual acuities of $6 / 5$ or better in the affected eyes and they produced abnormal low contrast visual acuity scores. However, scores from eyes with CMV retinitis were not analysed statistically due to the small number of the sample.

The type of HIV infection was classified according to the Centre for Disease Control (CDC) Classification of HIV Infection. Figure 2 shows the results of this classification for the population tested.

The results of the low contrast visual acuities of the control group, the 23 HIV positive patients without any ophthalmoscopic abnormalities, and the eight patients with HIV retinopathy are shown in Figure 3.

\section{Centre for Disease Control (CDC) Classifiçation of HIV (+) Subjects}

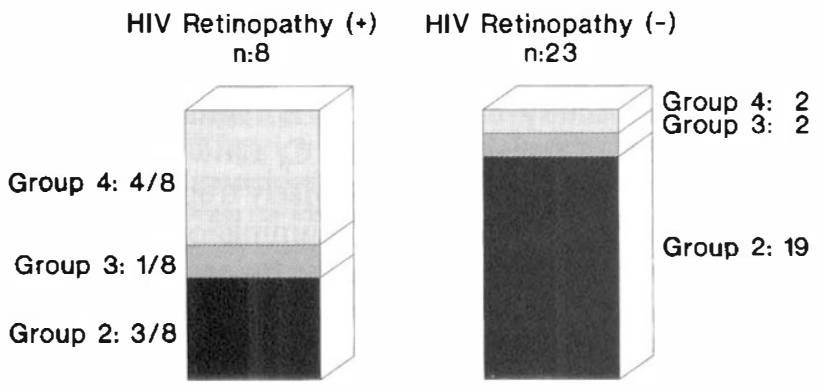

Grovo 1:Acute Seroconversion Grovo 2:Incubation Period

Groue 3: Symtomatic Group 4:Full Blown

Fig. 2. Distribution of the patients with or without visible ophthalmoscopic signs according to CDC Classification of HIV infection.
After the scores were adjusted for differences in Chart A scores between the controls and HIV sero-positive subjects, analysis of variance showed statistically significant reduction in Chart B and Chart $\mathrm{C}$ scores of the HIV seropositive subjects without retinopathy and HIV seropositives with retinopathy $(\mathrm{p}<0.01)$.

The results of receiver operating characteristic (ROC)

REGAN LOW CONTRAST VISUAL ACUITY CHART SCORES

HV SEROPOSTIVE PATENTS AND CONTROL SUBECTS

MEAN $\pm 2 S D$
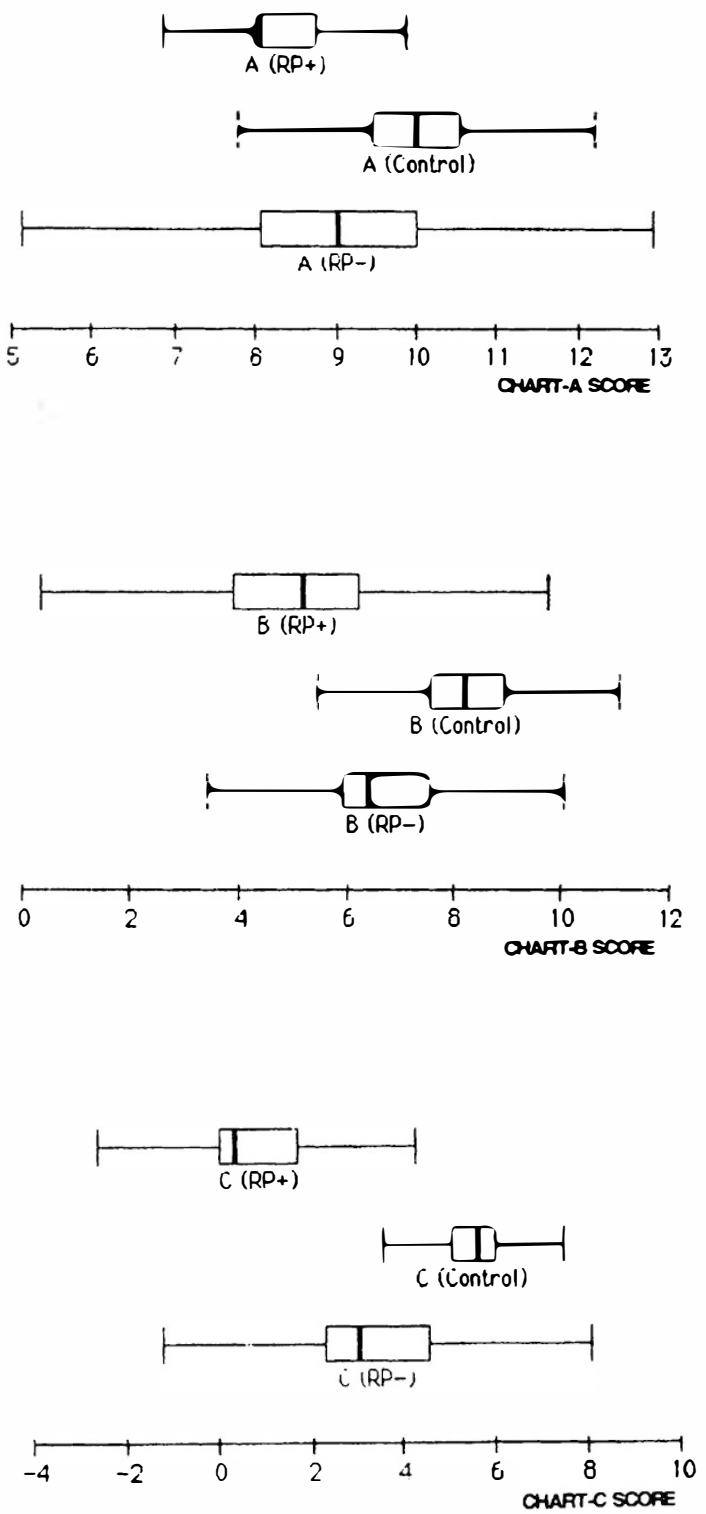

RP+ : HV RETMOPATHY PFESENT

PP. : NOFMAL FUNO

Fig. 3. Box-and-whiskers plot of the Chart $A, B$ and $C$ scores from HIV sero-positive individuals with retinopathy $(R P+)$, without retinopathy $(R P-)$ and age matched controls. 


\section{Regan Chart B}

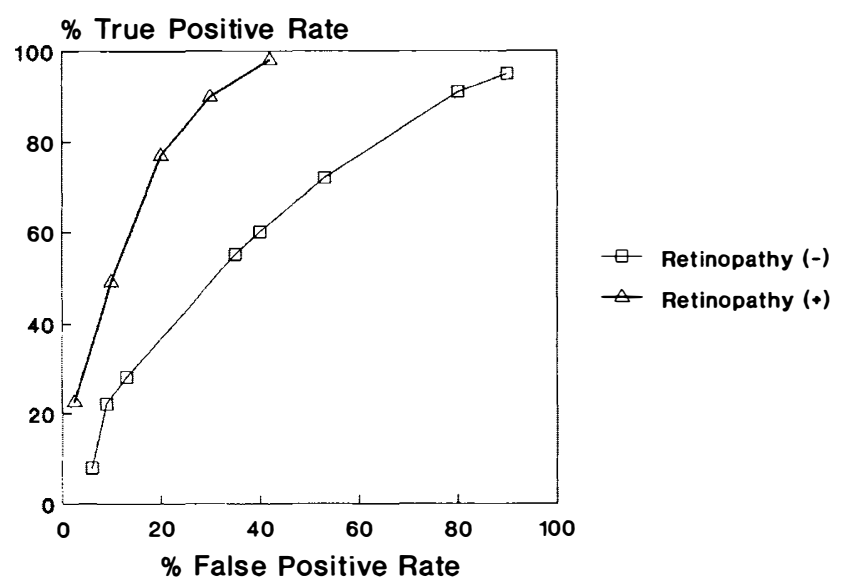

\section{Regan Chart C}

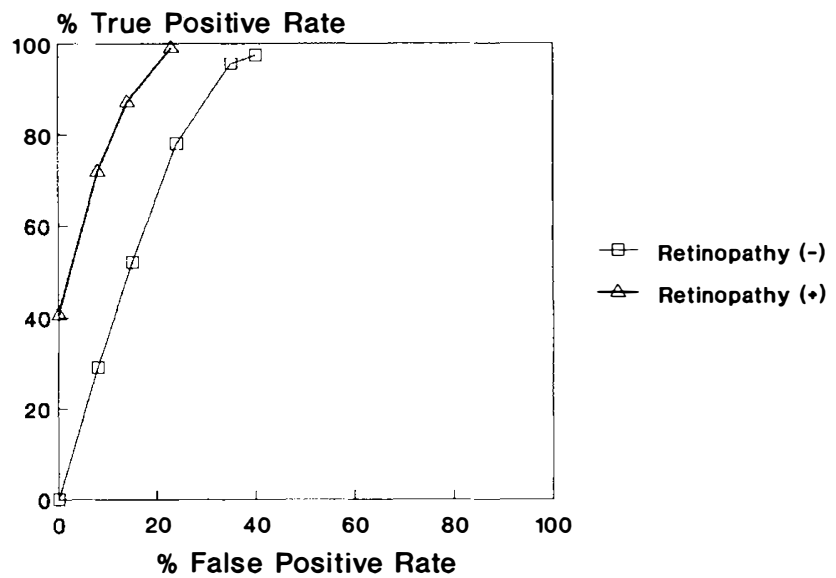

Fig. 4. Receiver operating characteristic (ROC) analysis of Chart $B$ and $C$ scores for HIV sero-positive individuals with or without HIV retinopathy (cotton-wool spots) and age-matched controls. True positive rate indicates the possibility of an abnormal test result in the disease category against the possibility of a false positive result in disease-free subjects at various cut-off points. Chart $C$ is particularly sensitive and discriminatory in detecting HIV (+) individuals with retinopathy.

analysis of Chart B and C scores for seropositive individuals with and without retinopathy are shown in Figure 4. Chart $\mathrm{C}$ was found to be particularly sensitive for detecting HIV positive individuals with or without retinopathy.

\section{DISCUSSION}

The most interesting finding of this pilot study is that HIV sero-positive individuals appear to have reduced low contrast acuities despite normal looking fundi. The level of decrease in low contrast visual acuities in eyes with HIV retinopathy was more pronounced. Two AIDS patients were seen with severe visual acuity loss, constricted visual fields and vitritis. However, they had normal retinal appearances and retinal dysfunction preceded the development of widespread funduscopic abnormalities. These patients have been reported previously and it was suggested that this dysfunction may represent the ophthal- mic equivalent of HIV encephalitis. ${ }^{9}$ We did not perform fluorescein angiography in our subjects and it is possible that retinal microcirculatory abnormalities might be present despite clinically normal appearance.

The effect of human immunodeficiency virus (HIV) on the central nervous system (CNS) is profound and widespread. Cerebral pathological changes are found in $75 \%-90 \%$ of individuals with acquired immune deficiency syndrome who are subsequently examined at autopsy. ${ }^{10.11}$ HIV has been detected in macrophages, glial cells and neurons in the CNS. ${ }^{12-17}$ Thus neurological dysfunction may arise not only from opportunistic infection, but also from primary infection of the central nervous system with HIV and consequent subtle demyelination and neurotrophic dysfunction. However, neurological symptoms (AIDS Dementia Complex = HIV Encephalopathy as the commonest) may be too subtle to be identified, leading to underestimation of the prevalence of AIDS-related central nervous system involvement. ${ }^{13,14}$

Therefore, low contrast visual acuity suppression in $\mathrm{HIV}(+)$ patients in the absence of any refractive error and visible ophthalmoscopic signs may reflect otherwise subclinical CNS involvement in HIV infection.

Inflammatory and ischaemic optic neuropathy has been reported in AIDS. ${ }^{18.19 .20}$ However, we are unaware of any previous report indicating subclinical optic nerve and/or visual system dysfunction due to CNS involvement in HIV infection.

Non-infectious HIV retinopathy (cotton-wool spots) occurs in $60-70 \%$ of the cases, usually alone or with CMV retinitis. ${ }^{20,21,22}$ The incidence of HIV retinopathy which has been of uncertain prognostic significance in HIV infection until recently, increases with increasing severity of HIV infection since T-helper (CD4) to suppressor (CD8) cell ratio significantly associates with HIV retinopathy and the ratio has been found to be lower when retinopathy is present even in the absence of any other symptoms of HIV infection. ${ }^{23}$ On the other hand, CMV retinitis which is the commonest ocular infection in AIDS frequently affects the peripheral retina as the initial site of the infection without any visual acuity change..$^{20.21,22}$ It has been suggested that the ophthalmologist may play an important role in early diagnosis of HIV infection since non-infectious retinopathy and intraocular opportunistic infections are the first presenting signs of the disease in many cases. ${ }^{24}$

In order fully to document the diagnostic value of such charts in HIV infection, we are prospectively studying long-term contrast sensitivity function changes in larger subgroups of patients in combination with detailed neuropsychiatric assessment, audio-evoked potential measurements and post-mortem neuropathological examination of the visual pathways.

This study was supported by a grant from W. H. Ross Foundation for Prevention of Blindness, Scotland.

The authors are indebted to Drs. Greame Mackintosh, Brian Fleck and Gordon Dutton for their assistance with the equipment, clinical arrangements and manuscript. 
Key words: Contrast Sensitivity, Visual Acuity, Low Contrast Visual Acuity, HIV, AIDS, Retinopathy

\section{REFERENCES}

1. Holland GN: Ophthalmic disorders associated with the acquired immune deficiency syndrome. In: Insler MS, ed. AIDS and other sexually transmitted diseases and the Eye. Orlando: Grune and Stratton Inc. 1987. Chapter 7.

2. Regan D and Neima D: Low contrast letter charts in early diabetic retinopathy, ocular hypertension, glauncoma and Parkinson's disease. Br J Ophthalmol 1984, 68: 885-9.

3. Bodis-Wolner I: Visual acuity and contrast sensitivity in patients with cerebral lesions. Science 1972, 178: 769-71.

4. Regan D and Neima D: Low contrast letter charts as a test of visual function. Ophthalmology 1983, 90: 1192-200.

5. Lorance RW, Kaufman D, Wray SH, Mao C: Contrast visual testing in Neuro-Visual Diagnosis. Neurology (Cleveland) 1987, 37: 923-9.

6. Drucker MD, Savino PJ, Sergott RC, Bosley TM, Schatz NJ, Kubilis PS: Low contrast letter charts to detect subtle neuropathies. Am J Ophthalmol 1988, 105: 141-5.

7. Regan D, Raymond J, Gingsburg A, Murray TJ: Contrast sensitivity, visual acuity and the discrimination of Snellen letters in Multiple Sclerosis. Brain 1981, 104: 333-50.

8. Warman RR and Glaser JS: Comparison of optotype contrast sensitivity and visual evoked potentials in optic nerve disease. Neuro-ophthalmology 1989, 9: 195-202.

9. Brodie SE and Friedman AH: Retinal dysfunction as an initial ophthalmic sign in AIDS. Br J Ophthalmol 1990, 74: 49-51.

10. Navia BA, Cho ES, Petito CK, Price RW: The AIDS dementia complex: II. Neuropathology. Ann Neurol 1986, 19: 525-35.

11. Nielsen SL and Davis RL: Neuropathology of Acquired Immune Deficiency Syndrome. In: Rosenblum ML, Levy RM, Bredesen DE, eds. AIDS and the Nervous System. New York: Raven Press, 1988; Chapter 8.

12. Price RW, Sidtis JJ, Navia BA, et al.: The AIDS Dementia Complex. In: Rosenblum ML, Levy RM, Bredesen DE, eds. AIDS nad the Nervous System. New York: Raven Press, 1988; Chapter 10.
13. Koenig S, Gendelman HE, Orenstein JM: Detection of AIDS virus in macrophages in brain tissue from AIDS patients with encephalopathy. Science 1986, 233: 1089-93.

14. Pumarola-Sune T, Navia BA, Cordon-Cardo C: HIV antigen in the brains of patients with the AIDS dementia complex. Ann Neurol 1987, 21: 490-6.

15. Gyorky F, Melnick JL, Gyorkey P: Human immunodeficiency virus in brain biopsies of patients with AIDS and progressive encephalopathy. J Infect Dis 1987, 155: 870-6.

16. Apatoff BR, Lee MR, Gurney ME: Trophic effects of neuroleukin on central neurons and functional interactions with HIV envelope protein. Ann Neurol 1987, 22: 156.

17. Levy RM and Bredesen DE: Central nervous system dysfunction in Acquired Immune Deficiency Syndrome. In: Rosenblum ML, Levy RM, Bredesen DE, eds. AIDS and the Nervous System. New York: Raven Press, 1988; Chapter 3.

18. Winward KE, Hamed LM, Glaser JS: Spectrum of optic nerve disease in human immunodeficiency virus infection. Am J Ophthalmol 1989, 107: 373-80.

19. Brack MJ, Cleland PG, Owen RI, Allen ED: Anterior ischaemic optic neuropathy in the acquired immune deficiency syndrome. Br Med J 1987, 295: 696-7.

20. Jabs DA, Green WR, Fox R: Ocular manifestations of acquired immune deficiency syndrome. Ophthalmology 1989, 96: 1092-9.

21. Jabs DA, Enger C, Bartlet JG: Cytomegalovirus retinitis and Acquired Immune Deficiency Syndrome. Arch Ophthalmol 1989, 107: 75-80.

22. Henderly DE, Freeman WR, Causey DM, Rao NA: Cytomegalovirus retinitis and response to therapy with ganciclovir. Ophthalmology 1987, 94: 425-34.

23. Freeman WR, Chen A, Henderly DE, Levine DM, et al.: Prevalence and significance of AIDS related retinal microvasculopathy. Am J Ophthamol 1989, 107: 229-35.

24. Freeman WR, Henderly DE, Lipson BK, Rao NA, Levine AM: Retinopathy before the diagnosis of Acquired Immune Deficiency Syndrome. Ann Opthalmol 1989, 21: 468-74. 